BONPLANDIA 20(2). 2011

ISSN: 0524-0476

\title{
REVISIÓN DE LA ETNOMEDICINA EN ARGENTINA: CONSTRUCCIÓN DE LA DISCIPLINA Y PERSPECTIVAS PARA EL FUTURO
}

\author{
CECILIA TRILLO¹, BÁRBARA ARIAS TOLEDO² \& SONIA COLANTONIO²
}

\begin{abstract}
Summary: Trillo, C., B. Arias Toledo \& S. Colantonio. 2011. A Review of the Ethnomedicine in Argentina: The construction of the discipline and perspectives for the future. Bonplandia 20(2): 405-417.

Ethnomedicine is a prolific field of study in Argentina. Different moments in the historic construction of the disciplines are clearly observed: the Hispanic-colonial period, with records mainly made by Jesuitical priests; a period between XIX and XX centuries, in which surveys with an ethnographic approach were made, registering in different languages the names of the plants and their associated uses by communities, which they were quickly disappearing; and finally, a change in investigation programs is registered. Modern surveys get deeper in an anthropological approach, including ecology, new disciplines like urban Ethnomedicine appear, as well as useful contributions to decision make about natural resources management for conservation. Finally, it is suggested that future studies include ecological issues, phytochemical, pharmacological and toxicological assessment of the species, and the strategies of use developed by communities, focusing in protection and conservation of species as well as human communities studied.
\end{abstract}

Key words: Ethnomedicine, medinal plants, Argentine.

Resumen: Trillo, C., B. Arias Toledo \& S. Colantonio. 2011. Revisión de la Etnomedicina en Argentina: construcción de la disciplina y perspectivas para el futuro. Bonplandia 20(2): 405417.

La Etnomedicina es un campo de investigación muy prolífico en Argentina. En el proceso histórico de construcción de la disciplina se observa claramente diferentes momentos: el período hispánico colonial con recopilaciones fundamentalmente a cargo de religiosos jesuitas; un período comprendido entre los siglos XIX y XX en el que se llevan adelante estudios de corte etnográfico registrando en las diferentes lenguas los nombres de las plantas y usos asociadas a ellas por parte de pueblos que rápidamente estaban desapareciendo, y finalmente se registra un cambio en los programas de investigación. Las investigaciones modernas profundizan aspectos más interpretativos de corte antropológico, incorporan miradas nuevas a partir de la ecología, aparecen nuevos estudios como la etnomedicina urbana y aportes necesarios para tomar decisiones en el manejo de recursos naturales para la conservación. Finalmente se sugiere que los estudios futuros incluyan los aspectos ecológicos, la evaluación fitoquímica, farmacológica y toxicológica de las especies, y por último, hacer énfasis en la protección y conservación, tanto de las plantas como de las comunidades humanas estudiadas.

Palabras claves: Etnomedicina, plantas medicinales, Argentina.

${ }^{1}$ Cátedra Diversidad Vegetal 11, FCEFyN. UNC.

${ }^{2}$ Cátedra de Antropología Biológica y Cultural, FCEFyN UNC. CONICET.

ceciliatrillo1@gmail.com 


\section{Introducción}

En los ambientes naturales y modificados por el hombres existe un amplio elenco de especies potencialmente medicinales disponibles e históricamente utilizadas por los pueblos. En Argentina la flora medicinal nativa reconocida consta de 1529 taxa (Barboza \& al. 2009), de las cuales solo una fracción ha sido estudiada en cuanto a sus efectos bioactivos, lo que no impide que su uso sea generalizado y vigente, principalmente en ámbitos rurales, pero también en las ciudades.

Sin bien el estudio de las plantas medicinales representa una parcialidad del conocimiento botánico tradicional, éstas constituyen valiosos elementos de análisis, a las que, en el proceso de curación se añaden elementos simbólicos, emocionales y mágico-religiosos, constituyendo un sistema etnomédico que ha sido profusamente estudiado por los especialistas argentinos.

La presente contribución realiza una revisión del elenco de publicaciones que se avocan al estudio de la farmacopea y etnomedicina argentinas. Si bien se pretende ser lo más exhaustivos posible, la presente revisión no constituye un relevamiento absoluto de lo publicado por autores argentinos en Etnomedicina, si no que busca señalar líneas temáticas destacadas y aspectos emergentes de lo publicado en la temática. Así, analizamos la información etnobotánica publicada para el período 1950-2011 con el fin de encontrar regularidades y analizar los ámbitos de estudios que quedan pendientes en los estudios científicos para el futuro. Para ello se revisaron publicaciones especializadas (nacionales e internacionales), bases de datos y documentos disponibles en la Web, agotando en éste último caso todo lo disponible en Economic Botany, Scripta Ethnológica, Journal of Ethnopharmacology, BLACPMA, Bonplandia, Darwiniana, The Open Complementary Medicine Journal, Parodiana, Dominguezia, Kurtziana, Rojaziana, Journal of Ethnobiology and Ethnomedicine. Cabe destacar que a fines evitar repeticiones, cuando mismos autores poseen más de una publicación de gran similitud en su contenido, se citó sólo una, privilegiándose las publicaciones nacionales.
La recopilación de información sobre plantas medicinales en culturas nativas latinoamericanas se remonta al período Hispánico Colonial. Ya en 1570 una Cédula Real firmada por Felipe 11 hace explícito el interés del estado en las plantas medicinales expresando que "...todas las hierbas, árboles, plantas, o semillas con cualquier valor medicinal que se pueda encontrar en aquellos lugares serán enviados a este Reino". Así, el primer proyecto para recoger información acerca de los conocimientos médicos de los nativos de forma sistemática comenzó en mayo de 1574 y fue publicado por la Corona española en 1597 bajo el título de "Introducción y memorias de la descripción de las Indias que su Majestad mandó hacer para el buen gobierno y ennoblecimiento dellas". Los registros, documentos y datos que aportaron los colonizadores, religiosos y exploradores que visitaron la región encontraron su fuente de información primaria en las tradiciones locales, incorporando luego el conocimiento aborigen respecto al uso medicinal de las plantas nativas (Cantero \& Ariza, 2006). En tal contexto, el conocimiento de los nativos fue reconocido como valioso cuando pudo ser "traducido", y el proceso de traducción implicaba primero el reporte de las tradiciones populares y leyendas. Posteriormente, los botánicos identificaban taxonómicamente la planta a partir de registros previos de otros botánicos y naturalistas que estudiaron la zona y, por último, vino el análisis y la caracterización química de los componentes, el cual constituyó un aporte más moderno a partir de los avances tecnológicos que se posee en la actualidad (Nieto, 2005).

Los primeros datos acerca de la flora medicinal útil del Río de la Plata, y su uso por parte de comunidades nativas, provienen de religiosos misioneros, entre los que se destacan los miembros de la Compañía de Jesús. En el marco de su tarea evangelizadora, los misioneros tomaban contacto con personas de diferentes etnias y mostraban especial interés por sus costumbres y conocimientos. Ejemplos de relatos sobre medicina tradicional de diferentes pueblos por parte de religiosos jesuitas son los trabajos de Pedro Montenegro (1945), Lozano, (1873), Sánchez Labrador 
(1910), Paucke (1944), Müller (1989) y especialmente los de Furlong (1969) en su relevamiento de la Historia social y cultural del Río de la Plata.1536-1810 el que, entre otros aspectos, sistematizó el aporte de los jesuitas en Sudamérica.

La llegada a Argentina de botánicos convocados por la Academia Nacional de Ciencias inicia un nuevo período con el desarrollo de la actividad científica. Entre 1872 y 1874, Paul Lorentz, botánico alemán y su ayudante J. Hieronymus recolectaron asiduamente material desde la provincia de Córdoba llegando hacia el norte hasta Bolivia. Hieronymus, interesado en los aspectos utilitarios de las plantas, aprovechó esta oportunidad de conocer la flora argentina para indagar a los lugareños sobre el uso y aplicaciones de la flora colectada, lo que resultó en la publicación de la obra Plantae Diaphoricae Florae Argentine en 1882 .A principios del siglo XX comienzan a aparecer destacados trabajos de académicos argentinos (Río \& Achával, 1905) y folkloristas (DiLullo, 1929, 1944,1946) que avanzaron en la recopilación de información acerca de las plantas, sus propiedades y usos.

Pero es recién en las últimas décadas del siglo $\mathrm{XX}$ en que se produce un salto cuali y cuantitativo al respecto. Es a partir de tal momento en que se focaliza la presente revisión, centrada en las publicaciones referidas a poblaciones de Argentina, debido a la dificultad de abarcar áreas mayores a causa de la vasta producción relativa a variados ambientes y a la diversidad cultural que en ellos se desarrolla. A la vez de considerar el aspecto histórico, la bibliografía relevada ha sido incluida y analizada considerando cuáles son los aspectos sobresalientes de la metodología particular empleada, agrupándose los de visión prioritariamente etnográfica, los que consideran aspectos ecológicos, aquellos que presentan evoluciones temporales o los que alertan sobre el riesgo de desaparición de especies y/o conocimientos, los que describen sistemas etnomédicos, aquellos que focalizan sobre características etnofarmacológicas de las especies, así como los que se vuelcan a la etnobotánica urbana.

Dentro del ámbito académico, los primeros aportes -pero que continúan hasta la actualidadson de corte etnográfico, proveyendo de relevamientos y descripciones acerca del uso de la flora por parte de diversas etnias aborígenes y que incorporan progresivamente elementos ecológicos al análisis. Se destaca, con el uso de metodología moderna etnográfica, Martínez Crovetto (1968a, 1968b,1981) con la exhaustiva tarea de entrevistar los últimos pobladores onas y araucanos y registrar los nombres, la fonética y los usos de las plantas, así como una revisión de las plantas medicinales utilizadas por comunidades guaraníes, entre otros aportes. Para las etnias del Gran Chaco fueron fundamentales los trabajos de Arenas y su grupo, pionero en la Etnobotanica argentina, tales como su estudio de la etnobotánica del grupo LenguaMaskoy (Arenas, 1981), la entrevista a Schulz sobre sus trabajos en la medicina del litoral (Schulz, 1997), la reseña de los aportes del padre Franz Müller (Arenas,1997), la etnobotánica de especies o grupos claves como la "doca" (Morrenia odorata) (Arenas, 1999), la "ruda" (Ruta chalepensis) (Arenas \& Galafassi, 1994), así como estudios sobre la farmacopea y la curación de enfermedades (Arenas, 2000) y Chifa \& Ricciardi (2001). Simultáneamente Filipov (1997) y Filipov \& Arenas (2008) aportó a este conocimiento con los estudios acerca de la farmacopea y sistemas etnomédicos de los pilagás de Formosa y la incorporación de los algunos vegetales a su farmacopea para el control de la fecundidad por el contacto con los criollos de la zona; y con los de la farmacopea toba para el cuidado y prevención de enfermedades bucales Martínez (2010 a). Últimamente la contribución de Scarpa (2009) con su trabajo acerca de los chorotes y los "préstamos" que ellos recibieron de las comunidades criollas que los rodean. En la misma zona chaqueña, Idoyaga Molina (1976, 1978, 1999) escribió sobre sexualidad reproductiva, gestación y gravidez entre culturas indígenas mataco y pilagás. En la Selva Misionera, los estudios de Crivos \& al. (2002) y Pocchetino \& al. (2007) en grupos Mbyá analizan el marco de sus construcciones simbólicas, mientras que en la Patagonia los trabajos de Conticello \& al (1997) y Bacigalupo (2001) analizan el 
uso de plantas medicinales en comunidades mapuches, tendencia que continúa con los trabajos de González \& Molares (2004), Lozada \& al. (2006), Estomba \& al. (2006) y Ladio \& al. (2007).

Paralelamente a los estudios anteriormente reseñados, hacia finales del siglo $\mathrm{XX}$ las temáticas tratadas, los grupos estudiados y las metodologías empleadas se diversifican exponencialmente. En relación a los nuevos grupos humanos estudiados comienzan a publicarse trabajos con "criollos", pobladores productos del mestizaje entre españoles y comunidades aborígenes en los que predomina la ocupación en la explotación agrícola ganadera de subsistencia, manufactura de tejidos y productos regionales que son trocados en los mercados o a la vera de los caminos junto con artículos de granja. Diversos autores sugieren una continuidad cultural en todo territorio del Chaco semiárido (Río \& Achaval 1905, Di Lullo 1946; Scarpa 2002, 2004) y para estepa patagónica Muiño (2010) y Ochoa \& al. (2010) que se observa en múltiples aspectos como el manejo del monte para la obtención de forraje y la curación con plantas medicinales y veterinarias, entre otros.

En la temática que nos ocupa se publican trabajos realizados con criollos ganaderos en Corrientes, tales como los de García (1984), Jiménez (1984) y García \& Jiménez (1986) quienes llevaron a cabo relevamientos de las plantas medicinales y las interrelaciones entre la magia y la medicina oficial, en el marco del mantenimiento de la salud por parte de estas comunidades rurales. En los trabajos posteriores, ya iniciado el siglo $\mathrm{XX1}$, se reflexiona sobre las transformaciones socioculturales experimentadas en las poblaciones ante los avances de la modernidad, se introducen aspectos ecológicos en los que se analizan factores ambientales, interpretaciones de corte antropológico y aportes a la posible gestión de recursos y conservación de ambientes. Así, encontramos los estudios efectuados con campesinos de Idoyaga Molina (2000) en Salta y Jujuy y los trabajos con criollos ganaderos en Formosa (Scarpa, 2002, 2004, 2005), en los que el autor describe los usos medicinales, sus clasificaciones y valoraciones. Para las etnias chaqueñas, se destacan las publicaciones de Arenas (2002, 2009), que realizan una muy interesante revisión histórica sobre los estudios centrados en las etnias del Gran Chaco. En las yungas, en la selva paranaense de Misiones, y en relación con ambientes andinos, aparecen los trabajos de Amat \& Yajía (1991), Hilgert \& Gil $(2006,2008)$ y Martínez \& al. (2004), revisando los usos medicinales y los cambios que se observan en las prácticas tradicionales y modernas de curación y mantenimiento de la salud. Mientras Keller y Romero (2006), en un estudio realizado con campesinos del área de la Reserva Yabotí destacan el amplio espectro de especies curativas tanto espontáneas como cultivadas, y reflexiona acerca de las oportunidades de realizar programas de diversificación rural sostenible basados en la predisposición de la comunidad para el cultivo y domesticación de múltiples especies, Zamudio \& al (2010) comparan el uso de mieles medicinales entre inmigrantes polacos y pobladores Criollos, señalando que no existen diferencias sustanciales en el corpus medico tradicional entre ambas poblaciones, a la vez la relación existente entre zooterapia y fitoterapia en ambas poblaciones. En el centro del país Martínez (2002), Martínez \& Planchuelo (2003), y Martínez \& al. (2004, 2009) analizan en comunidades criollas del Valle de Calamuchita los usos medicinales por parte de estos campesinos y la recepción de información sobre plantas medicinales en grupos de estudiantes, identificando especies claves para su conservación ante la presión de la recolección comercial. Arias Toledo (2006) y Arias Toledo \& al. (2007a, b) llevaron a cabo estudios sobre plantas alimenticias y medicinales en poblaciones del norte y oeste de la provincia de Córdoba, enfatizando en las relaciones con los diferentes ambientes. En dicha provincia Menseguez \& al. (2007) también realizaron aportes sobre el conocimiento y el uso de las plantas medicinales en el ámbito familiar y exploran algunos factores culturales (contacto frecuente con la ciudad, migraciones, etc.) que pueden influir en su utilización por parte de los pobladores de zonas rurales de Cerro Colorado; al tiempo que Bocco \& al (1997), Núñez \& Cantero (2000) y Goleniowsky \& al (2005) 
sistematizaron las plantas medicinales del sur de la provincia. Se suman a los anteriores los estudios sobre factores que afectan los mecanismos de transferencia y conservación del conocimiento etnobotánico efectuados por Lozada \& al. (2006), Eyssartier \& al. (2009) y Ochoa \& al. (2010) referidos a las culturas andino-patagónicas mestizas.

La aparición de publicaciones con claras referencias ecológicas se produce también en los años 2000. Al respecto pueden mencionarse las comparaciones entre localidades diferentes analizando factores modeladores ambientales y culturales. Algunos trabajos analizan diversos factores socioculturales y su estrecha interrelación con el conocimiento y el uso del ambiente (Arias Toledo 2009, Arias Toledo \& al., 2007a, 2009), mostrando que variables como la edad, el género, el nivel educativo y el origen contribuyen a modelar la relación de las personas con su ambiente, a la vez que otros autores analizan las relaciones entre los modos de vida y la medicina herbolaria (Hilgert \& Gill, 2008) mostrando que las especies utilizadas y los modos de uso varían en la medida que se abandonan prácticas tradicionales, en tanto que los estudios de Ochoa \& al. (2010) reflejan que es la autodefinición y la constitución como comunidad mapuche el principal factor que explica la mayor cantidad de conocimientos de especies medicinales nativas comparadas con los criollos andinos patagónicos. En cambio, serían principalmente los factores ambientales y las actividades rurales características del medio los que explicarían la prevalencia de determinadas enfermedades (Martínez \& al. 2004), tales como fracturas, desgarros, dolores reumáticos, "falseaduras", dolores óseos, y "tomar frío" en un medio hostil como los ambientes que se encuentran dentro de las ecorregiones del "monte", "puna". Lozada \& al. (2006) y Estomba \& al. (2006) han permitido evidenciar que los saberes sobre plantas de uso medicinal no se distribuyen homogéneamente en el espacio que los habitantes utilizan; los resultados demuestran que existen patrones diferenciales de uso entre ambientes, y denotan que para las especies de origen nativo se recorren mayores distancias para su recolección. Ello estaría relacionado con el valor cultural de estas plantas nativas, muchas de ellas de carácter irremplazable, las cuales fueron muy importantes en el pasado según los relatos de los habitantes del bosque de Cipreses, y que siguen estando vigentes entre los mismos. En la última década comienzan a desarrollarse programas de investigación en los que se analizan relaciones biogeográficas con énfasis en la utilización de plantas exóticas y nativas y que indagan sobre la incorporación de las nuevas especies medicinales que se introdujeron a nuestros ambientes por la conquista española y las posteriores inmigraciones europeas que llegaron a nuestro país. Tales investigaciones analizan el grado de apropiación eincorporación de las nuevas especies a la cultura ancestral y las prácticas de recolección y cultivo actuales que realizan los pobladores a fin de satisfacer sus necesidades. Para los habitantes rurales sería crucial la conservación y mantenimiento de la diversidad biológica y cultural propias de las tradiciones mapuches, a través de planes de manejo de los recursos naturales de la zona. Asimismo, Eyssartier \& al. (2009), en su investigación sobre huertos en comunidades semi-rurales patagónicas de ascendencia mapuche muestra que el uso de plantas medicinales cultivadas es una costumbre importante entre ellos. Las autoras sostienen que el notable poco uso de especies nativas sugiere que el conocimiento ancestral está sufriendo procesos de aculturación asociados a profundas transformaciones ocurridas en estas poblaciones, relacionadas en parte con una fuerte influencia occidental ejercida desde la medicina oficial, así como la intervención de agentes de extensión. Ochoa \& al. (2010) señalan que los pobladores auto-reconocidos y organizados en la comunidad mapuche poseen un conocimiento más profundo que los criollos acerca de la flora medicinal silvestre en general, y de la nativa en particular. Señalan asimismo que en las comunidades mapuches el hecho de mantenerse organizados y en continuidad con una identidad cultural en la que las plantas históricamente ocuparon un lugar primordial ha favorecido el mantenimiento del saber sobre especies medicinales. También en otros ambientes se registran los mismos patrones, y autores como Hilgert \& Gill (2006) ponen de manifiesto que las plantas nativas 
y exóticas se utilizan de manera diferencial según el sistema etnomédico elegido: son mayores las menciones de nativas para enfermedades "culturales" o "sobrenaturales". Para entender estos registros, consideran dos aspectos a analizar de naturaleza diferente: uno relacionado con la escasez de ambientes en buen estado de conservación donde encuentran las especies nativas y el acceso asegurado a zonas degradadas con abundantes especies asilvestradas como malezas y, por el otro, la adaptación cultural de incorporar nuevas especies exóticas con altos niveles de valoración por parte de la comunidad como producto del sincretismo a lo largo del tiempo.

Por otro lado, y en la medida en que existen registros escritos de muchos años de antigüedad, es posible analizar el uso de las especies medicinales desde una perspectiva histórica, buscando referencias de sus usos en las fuentes bibliográficas actuales y del pasado (las cuales pueden no obedecer a una metodología etnográfica). Estas comparaciones arrojan resultados coincidentes y abren nuevos interrogantes sobre los mecanismos de transmisión del conocimiento. Para tal análisis se cuenta con los trabajos de Hilgert \& Gill (2006) que en sus estudios sobre la riqueza de especies medicinales y prácticas de curación utilizadas por ganaderos de las yungas argentinas, mencionan 259 spp que representan entre el 70 y el $80 \%$ del total de estimado y el uso de tradiciones etnomédicas, a pesar de disponer de centros de salud oficial y medicinas de origen industrial. En la misma línea, las investigaciones realizadas en pobladores rurales de la provincia de Córdoba por Trillo \& al. (2010) registran el uso de 153 spp. que representan más del $85 \%$ del total de plantas medicinales registradas hace más de 100 años en la bibliografía folklórica y científica, revelando la persistencia de un complejo sistema etnomédico. Este último incluye múltiples prácticas de la medicina tradicional como el uso de cintas, los rezos a Santos y Vírgenes, las oraciones propiciatorias, el "curanderismo" y "el autotratamiento por legos".

Sin embargo, y aún cuando hay consenso acerca de la vigencia de sistemas etnomédicos tradicionales, existe urgencia por registrar el conocimiento y uso popular de diferentes especies, debido a los complejos cambios ambientales y socioculturales a los que se está asistiendo. Diferentes autores aportan trabajos que reflejan estos cambios rápidos, nuevos y que ponen en riesgo los estilos de vida de comunidades enteras. Así, se presentan estudios realizados entre aborígenes mbya y colonos misioneros, afirmando que la pérdida del monte como fuente de recursos, consecuencia de la progresiva deforestación de la selva, resulta responsable en parte de la pérdida de las condiciones fundamentales propias de las estrategias de vida en el área rural (Crivos \& al. 2005). Martínez (2005), propone en su estudio sobre recolección, comercialización y conservación de plantas medicinales que no es posible afirmar que la recolección sea sustentable en todos los casos, y conjuntamente con otros autores (Martínez \& al., 2006 y Martínez \& al., 2009) confirman la necesidad de enfatizar la tarea de conservación y de extender investigaciones de este tipo a especies que deberían ser prioridad en programas de conservación. Con objetivos similares pero en la selva paranaense Moreau (2009)analiza la problemática dellevaradelante esfuerzos de conservación de conocimiento y ambientes. Finalmente, Arias Toledo \& al. (2010) plantean la problemática referida a la pérdida de conocimiento etnomédico cuando desaparecen los bosques nativos y el simultáneo avance de la frontera agrícola, que impacta sobre el ambiente natural y sobre la cultura de los pobladores a través de la erosión del conocimiento tradicional. Proponen que la desaparición del bosque hace desaparecer de la memoria colectiva de pobladores las formas de manejo tradicionales, posiblemente más sustentables o menos lesivas que el monocultivo industrial, y destacando que tal pérdida ambiental y cultural agrava tanto la situación ambiental de la región como la situación socioeconómica de los pobladores.

Sin lugar a dudas, algunos de los aportes más ricos y completos son los relacionados a los sistemas etnomédicos dado que, en búsqueda de la comprensión de los numerosos matices -incluyendo aspectos sagrados en la medicina-, han sidoampliamente estudiados por 
antropólogos y etnobotánicos en todo el país. Comienzan destacándose las publicaciones de Dasso (1985) e Idoyaga Molina (2000, 2001) quien refleja "lo sagrado" en las prácticas terapéuticas de los curanderos y detalla que, para el usuario, el curandero no sólo posee más conocimiento que los profesionales médicos sino que además posee más poder, en relación a lo sagrado, pues es asistido por los santos y vírgenes auxiliares, además de hacer presente el Dios cristiano.

Múltiples autores registran en diferentes grupos humanos la riqueza de matices emocionales, religiosos y sagrados que explican los procesos de curación: entre ellos los aportes de Gonzalo (2002), Martínez \& Planchuelo (2003), Arias Toledo (2006), Hilgert \& Gill (2006), Martínez (2007abc), Menseguez \& al. (2007), Martínez (2008ab), Hilgert (2001,2009), Martínez \& al. (2009), Martínez (2010 b). Particularmente en el noreste de Argentina, Remorini \& Sy (2002), realizan aportes a la caracterización etnográfica de representaciones y prácticas en torno a la salud y la enfermedad de los Mbya de la provincia de Misiones, exploran acerca de la etiología de la enfermedad enfocando el universo de creencias, en el que la concepción de bienestar y salud están asociados a un estado de equilibrio y armonía con el entorno. Los autores relatan que dicho equilibrio depende de pautas culturales ancestrales, cuyo incumplimiento se traduce en un desajuste en la relación con el medio y en el normal funcionamiento del organismo humano generando enfermedad, muerte y otros males, conclusiones similares a las arribadas por Crivos \& al. (2006) en su estudio sobre el origen y función de los parásitos en dos comunidades Mbyá de Misiones. Análisis muy detallados sobre la etnomedicina de los criollos del noroeste son llevados a cabo por Scarpa (2002), con estudios sobre la farmacopea utilizada en los trastornos digestivos, así como un análisis del "síndrome cálido-fresco" (Scarpa, 2005) que constituye el criterio etiológico-terapéutico central de la medicina popular criolla. Otro exhaustivo examen de la entidad nosológica conocida como "mala sangre" también fue realizado por Scarpa ( 2008); tal entidad constituye tanto una enfermedad folk en sí misma como la explicación etiológica de numerosos trastornos que responde a variados agentes que, como denominador común, tienden a alterar las condiciones normales, necesarias para un buen funcionamiento del organismo. En el Noroeste argentino Hilgert \& Gil (2007) comparan los sistemas tradicional e institucional de medicina en relación a la salud reproductiva, señalando numerosos aspectos mágico-simbólicos y rituales en la concepción popular de los aspectos reproductivos, que deberían ser comprendidos por la medicina institucional para lograr la incorporación de los pobladores a las prácticas sanitarias oficiales.

Un aspecto poco tratado en la Etnobotánica argentina es el de las características etnofarmacológicas de las plantas medicinales. Al respecto, Molares \& Ladio (2008) analizan la clasificación y utilización de las especies curativas en relación con la presencia de propiedades organolépticas en la comunidad mapuche patagónica. Las autoras detectan que la mayor parte de las 145 etnoespecies medicinales son plantas con aroma y/o sabor $\mathrm{y}$, en menor medida, con morfologías y texturas distintivas. Ello, queda expreso en el uso indistinto de las palabras "gusto" y "olor", y lo confirman estadísticamente con la correlación positiva entre ambos, a la vez que el color de las preparaciones líquidas también fue en el set de respuestas sobre características organolépticas. Sugieren así que la separación entre sabor y aroma parece ser más una construcción teórica del pensamiento occidental que una actitud cultural o biológica, y concluyen que la información obtenida se encuadraría en la "teoría de signaturas" según la cual las plantas enteras, sus partes o sus extractos llevan el signo de sus virtudes terapéuticas o simbólicas inscrito en su propio aspecto. Keller (2007), trabajando con Mbya y Ava Chiripa también refleja el uso de la doctrina de la signatura a la vez de visiones chamánicas y criterios asociativos en base a plantas similares como criterios de selección de plantas medicinales. Por su parte, Barboza \& al. (2009) presentan un catálogo de la flora medicinal nativa de Argentina, incluyendo la composición química y actividad biológica de 
las especies para quienes tal información está disponible.

Finalmente, mientras que la mayor parte de las publicaciones en Etnobotánica se habían centrado en los pobladores de zonas rurales, existen autores que deciden explorar cuál es la relación de los habitantes citadinos con las plantas. En las grandes ciudades los pobladores no tienen acceso, o el mismo es dificultoso, a ambientes naturales para aprovisionarse de plantas medicinales y deben recurrir a los comercios que se las ofrecen, por cuanto resultan de particular interés los estudios acerca de la comercialización y control de calidad de los productos elaborados. Existen estudios como los de Cambi \& al (1999) y Herman $\&$ al (2001) que registran altos consumos de especies pero escaso reconocimiento de las especies a campo y desconocimiento sobre los problemas por el incorrecto uso. Los autores alertan sobre situaciones de sustitución y adulteración de drogas genuinas (codificadas en la Farmacopea Nacional Argentina) tanto como errores en la identidad botánica de otras plantas no oficiales (las que aún no han sido codificadas en la FNA), y sobre la necesidad de que los organismos de control actúen correctamente para la preservación de recursos resaltados por las OMS por su valor social, como lo son los fitoterápicos (Cuassolo \& al., 2009). En el litoral argentino el trabajo de Pirondo \& al (2011) alertan sobre la influencia de los factores externos relativos a la comercialización de plantas medicinales (acopiadoras, medios de comunicación, escenarios de ventas) como homogeneizadores del conocimiento de medicina popular. En Buenos Aires, Pochettino \& al. (2008) analizan las "dietéticas" de la ciudad de La Plata como el lugar privilegiado para el abastecimiento de alimentos saludables, suplementos dietéticos y materias primas para infusiones generalmente terapéuticas. El estudio refleja que los consumidores de productos elaborados generalmente poseen un conocimiento restringido a las propiedades del producto, menos frecuentemente a su composición y raramente al origen y modos de obtención y procesamiento de los componentes. Las autoras señalan, no obstante, que este conocimiento, aunque parcializado y fragmentario, no debe ser desdeñado, ya que constituye el motivo central que fundamenta el consumo de determinados productos en tanto se desechan otros. También en Buenos Aires, en un contexto periurbano de la ciudad de Tandil, Hilgert \& al. (2010), en la localidad de Hernández (Pochettino \& al, 1997) y en la Isla Paulino (Hernandez \& al, 2010) muestran la vigencia de la medicina folcklórica que se complemente con la formal. Hilgert \& al. (op. cit), particularmente, señalan que huertas y baldíos se constituyen en sitios de gran importancia para la obtención de especies medicinales, las que se complementan con compras en comercios. En Bariloche, Cuassolo \& al. (2009) estudian los centros de expendio de plantas medicinales, considerados como contextos de suma importancia en los ambientes urbanos debido al valor otorgado a las medicinas de origen vegetal por la OMS en 1987 (Directrices sobre la Conservación de Plantas Medicinales. OMS, IUCN, WWF); por constituirse en una alternativa terapéutica en la atención primaria de la salud, las herboristerías son lugares donde se genera un flujo de información que se transmite socialmente y forma redes de saberes que mantienen vigente conocimientos locales sobre las plantas medicinales. Las autoras alertan sobre que, ante la ausencia de controles por parte del Estado, estos centros necesitan de la labor responsable de sus expendedores en cuanto a una mayor verificación de los productos que comercializan y su estado sanitario. Por último interpretan que los estudios etnobotánicos urbanos en relación a estos centros son una herramienta para registrar el conocimiento tradicional en continuo proceso de cambio $\mathrm{y}$, por qué no, de fusión con las sociedades modernas. Del Vitto \& al. (1997) en la provincia de San Luis registran 273 especies y toman en consideración que muchas de las hierbas medicinales regionales son poco conocidas desde los puntos de vista botánico, fitoquímico y farmacológico. Por ello, investigadores locales han iniciado estudios de esa naturaleza sobre dichas hierbas, en consonancia con el progreso en el conocimiento de la flora regional. Los autores proponen que, a raíz de la actual y acelerada regresión de las poblaciones naturales de 
estas plantas, resulta pertinente remarcar la necesidad y urgencia de dar protección a las plantas medicinales regionales, así como intentar sistemáticamente su introducción al cultivo, puesto que constituyen un invalorable recurso natural que aceleradamente está siendo llevado a la extinción.

\section{Discusión}

En principio, el tipo de análisis de "series de tiempo", en el que se realizan relevamientos históricos a lo largo de un período de tiempo, es una metodología vigente en estudios etnobotánicos que permite llevar adelante el análisis de la persistencia o la pérdida de información que experimentan los pobladores rurales. La evidencia de la presencia de una tradición etnomédica tan profunda y antigua es una de las explicaciones que permiten entender cómo estas comunidades tradicionales siguen conservando prácticas y conocimientos, a pesar de los notables cambios socioeconómicos que las influenciaron durante las últimas décadas (Trillo, 2010).

Asimismo, las revisiones históricas en un área de estudio posibilitan visualizar las tendencias de la disciplina y los nuevos ámbitos de interrelación; en este caso, por ejemplo, con la ecología, con la biología de la conservación, la antropología, la fitoquímica, etc. La presente revisión arroja como dato emergente la progresiva incorporación de nuevos elementos de análisis, tales como aspectos ecológicos, o la inclusión de nuevos sitios o grupos de estudio, mostrando el creciente interés que la disciplina despierta entre los investigadores argentinos. Similares análisis fueron realizados en otros países latinoamericanos, mostrando tendencias comparables. Para Perú (La Torre Palacios, 2007), se centra en los estudios de las etnias amazónicas en el último siglo, y destaca que se observa una transición desde los temas lingüísticos hacia temas ecológicos a la vez que se incrementa el número de publicaciones, y explica tales sucesos a través de la prevalencia de las agendas ambientales y en base a la disminución de la cantidad de individuos de las etnias estudiadas, ubicación geográfica y preservación cultural. En el caso de la Etnomedicina brasileña, Calixto (2005) destaca el gran progreso en el número experimentado por las publicaciones en las últimas décadas.

Por otra parte, el análisis de las investigaciones llevadas a cabo hasta ahora nos permite reflexionar sobre cuáles son los aspectos más estudiados y sobre las temáticas o miradas que, como comunidad científica, nos queda por resolver en el futuro. En concordancia con lo apuntado por Cordell (2007) y Bermúdez \& al. (2005), consideramos que resulta de gran utilidad que los estudios en Etnobotánica médica incluyan los aspectos ecológicos, la evaluación fitoquímica, farmacológica y toxicológica de las especies medicinales identificadas, y las estrategias de uso que desarrollan las comunidades. Muy especialmente deben prevalecer criterios de protección y conservación, tanto de las especies como de las comunidades humanas estudiadas, utilizando el enorme potencial de las disciplinas etnobiológicas para el desarrollo de programas de conservación.

\section{Agradecimiento}

Agradecemos la generosa colaboración de los revisores del manuscrito y al Dr Gustavo J. Martinez por compartir con las autores publicaciones.

\section{Bibliografía}

AMAT, A. \& M. YAJIA. 1991 Plantas Medicinales y Etnofarmacología en la Provincia de Misiones (Argentina). Acta Farm. Bonaerense 10: 153-9.

ARENAS, P.1981.Etnobotánica Lengua-maskoy. Ed.Fundación para la educación, la ciencia y la cultura. Buenos Aires.

_ 1997. Nota introductoria al estudio del padre Franz Mûller sobre la farmacopea guaraní. Parodiana 10 (1-2):189-196, Buenos Aires.

. 1999. Morrenia odorata (Asclepiadaceae), an edible plants of the Gran Chaco. Econ. Bot. 53(1): 89-97.

—. 2000. Farmacopea y curación de enfermedades entre algunas etnias del Gran Chaco. En: A.G. 
Amat (Coord.) Farmacobotánica y Farmacognosia en Argentina (1980-1998) pp. 87-118. Ediciones Científicas Argentinas, La Plata.

- 2002. Historia sobre los estudios sobre plantas medicinales en el Gran Chaco. Disertación, Libro de Resúmenes, XXII Encuentro de Geohistoria Regional y I Simposio sobre el Estado Actual del Conocimiento Antropológico del Gran Chaco Meridional, Resistencia.

. 2009. Los estudios sobre medicina y farmacopea vernácula en el Gran Chaco. Rojasiana 8: 81-100

\& G. GALAFASSI. 1994. La Ruda (Ruta chalepensis L.-Rutaceae-) En la medicina folclórica del norte argentino. Dominguezia vol.11- $\mathrm{N}^{\mathrm{o}} 1$, Buenos Aires.

ARIAS TOLEDO, B. 2006 Aspectos cuantitativos, cualitativos y simbólicos de la medicina tradicional de los pobladores criollos de Cerro Colorado (Córdoba, Argentina). PINACO - Investigaciones sobre Antropología Cognitiva IV: 105-115.

a __ COLANTONIO, S.E. \& GALETTO, L. 2007 Knowledge and use of food and medicinal plants in two populations from the Chaco, Córdoba province, Argentine. Journal of Ethnobiology 27(2) 218: 232

b__ GALETTO, L. y COLANTONIO, S. 2007. Uso de plantas medicinales y consumo de alimentos silvestres según características socio-culturales en la Comuna de Los Aromos (Córdoba). Kurtziana 33 (1): 79-88.

- 2009. Diversidad de usos, prácticas de recolección y diferencias según género y edad en el uso de plantas medicinales en Córdoba, Argentina. BLACPMA 8(5): 389-401.

; GAletto, L. \& COLANTONiO, S. 2009. Ethnobotanical knowledge in rural communities of Córdoba (Argentine): the significance of cultural and biogeographical factors. Journal of Ethnobiology and Ethnomedicine 5:40-48.

_- TRILLO, C. \& GRILLI, M. 2010. Uso de plantas medicinales en relación al estado de conservación del bosque en Córdoba, Argentina. Ecología Austral 20:235-246.

BACIGALUPO, A.M.2001. El rol del medicinar de Machi en la recreación de la identidad mapuche: voces de resistencia, hibridez y transformación en las prácticas de machi. Scripta Ethnológica volumen XX11l, pp 89-119. Centro Argentino de Etnología Americana, Buenos Aires.

BARBOZA, G. E., J. J. CANTERO, C. NUÑEZ, A. PACCIOARONI \& L. ARIZA ESPINAR. 2009. Plantas medicinales: Revisión y "screening" fitoquímico y etnofarmacológico de la flora nativa de Argentina. Kurtziana 34 (1-2): 7-365.

BERMÚDEZ, A., OLIVEIRA-MIRANDA,M.A. \& D. VELAZQUEZ, 2005. La investigación etnobotánica sobre plantas medicinales: Una revisión de sus objetivos y enfoques actuales. INCI. (on line) vol.
$30, n^{\circ} 8: 453-459$.

BOCCO, E., N. VISCI Y N. MONTANI. (1997). Relevamiento de las plantas medicinales espontáneas del Departamento Rio Cuarto (Córdoba, Argentina). Parodiana 10: 11-18.

CALIXTO, J .2005. Twenty-five years of research on medicinal plants in Latin America. A personal view. Journal of ethnopharmacology 100:131-134

CAMBI, VN, HERMANN PM, BONTI EE, HAUSSAIRE MF, SÁNCHEZ DH. 1999. The use of medicinal plants in Bahía Blanca, Argentina. Acta Hortic. 501:57-61.

CANTERO, J.J. \& L. ARIZA ESPINAR. 2006. Breve reseña histórica sobre el uso de las plantas medicinales en la Provincia de Córdoba. En: Barboza, G. E., J.J.Cantero, C.O. Nuñez, y L.Ariza Espinar (Eds). 2006. Flora Medicinal de la Provincia de Córdoba. Pteridófitas y Antofitas silvestres o naturalizadas. Pp 2-13. Museo Botánico de Córdoba, Argentina.

CONTICELLO, L., R. GANDULIO, A. BUSTAMANTE Y C. TARTAGLIA (1997). El uso de plantas medicinales por la comunidad mapuche de San Martín de los Andes, Provincia de Neuquén (Argentina). Parodiana 10:165-180.

CORDELL, G. 2007. A vision for medicinal plants. BLACPMA 6 (4), 89 - 91.

CRIVOS, M., MARTINEZ, M.R,NAVONE, G., POCCHETINO,M.L. ARENAS,P. DIGIANI,M. TEVES,L. REMORINI,C.,SY,A.ILLKOW,C.\& DELORENZI,N. 2002.Un enfoque etnográficobiológico en el estudio de los enteroparásitos en comunidades Mbyá-Guaraníes (Misiones, Argentina). Sztuka Leczenia 8: 199-213.

, M. R. MARTÍNEZ \& M. L. POCHETTINO. 2005. La narrativa acerca del paisaje y del cambio ambiental entre los pobladores del área rural del municipio de Aristóbulo del Valle (Misiones, Argentina). Actas Congreso Argentino de Inmigración y IV Congreso de historia de los pueblos de la provincia de Santa Fe, Argentina.

—, M. R. MARTÍNEZ, M. L. POCHETTINO, L. TEVES, C. REMORINI \& A. SY. 2006. Los tachó. Consideraciones sobre el origen y función de los parásitos en dos comunidades Mbyá de la provincia de Misiones, Argentina. Scripta Ethnologica 28: 9-19.

CUASSOLO, F., A. LADIO, \& C. EZCURRA. 2009 Aspectos de la comercialización y control de calidad de las plantas medicinales más vendidas en una comunidad urbana del NO de la Patagonia Argentina. BLACMA 9 (3): 166-176.

CHIFA, C. y A. I. A. RICCIARDI 2001. Plantas de uso en medicina vernácula del Centro del Chaco Argentino. Miscelánea 117, Fundación Miguel Lillo, Tucumán, 34 pp.

DASSO, M.C. 1985. El shamanismo de los mataco de la margen derecha del Río Bermejo (Provincia del 
Chaco, República Argentina). Scripta Ethnologica 5: 9-35.

DEL VitTO, L. A., E.M. PETEnAtTI Y M.E. PETENATTI.1997. Recursos herbolarios de San Luis (República Argentina) Primera parte: plantas nativas. Multiquina 6: 49-66.

DI LULLO, O. 1929. La medicina popular de Santiago del Estero. Talleres gráficos El Liberal, Santiago del Estero.

_. 1944. El folklore de Santiago del Estero. Medicina y alimentación. Ed. El Liberal, Santiago del Estero, $431 \mathrm{pp}$.

—. 1946. Contribución al estudio de las Voces Santiagueñas. Imprenta López. Buenos Aires.

ESTOMBA, D., LADIO, A.H. \& LOZADA. 2006. Medicinal wild plant knowledge and gathering patterns in a Mapuche community from Northwestern Patagonia Journal of Ethnopharmacology 103:109-119

EYSSARTIER, C. LADIO, A. H \& LOZADA, M. 2009. Uso de plantas medicinales cultivadas en una comunidad semi-rural de la estepa patagónica. BLACMA 8 (2): 77 - 85.

FILIPOV, A.1997. La farmacopea natural en los sistemas terapéuticos de los indígenas Pilagá. Parodiana 10(1-2):35-74.

FILIPOV, A. y P. ARENAS. 2008. Los vegetales en el ciclo reproductivo de la mujer pilagá (Chaco Central, Argentina). Suplemento Antropológico, Universidad Católica, Asunción 43(2): 211-236.

FURLONG, G. 1969.Historia social y cultural del Río de la Plata, 1536-1810.El transplante cultural. Tipográfica Editora Argentina, Bs As.

GARCIA, S.P. 1984. Conocimiento empírico, magia y religión en la medicina popular de los Departamentos de Esquina y Goya (Corrientes). En: Cultura tradicional del área del Paraná Medio. Buenos Aires: Fundación Federico G. Bracht Editores. Instituto Nacional de Antropología.

— \& JIMÉNEZ, D. 1986. Natural y postizo: frío y caliente. Suplemento Antropológico 21. Universidad Católica, Asunción.

GOLENIOWSKI, M., BONGIOVANNI, G. A., PALACIO, L., NUÑEZ, C. O. \& CANTERO, J. J.2006. Medicinal plants from the "Sierra de Comechingones", Argentina. Journal of Ethnopharmacology 107: 324-341.

GONZALEZ, S. \& S. MOLARES. 2004. Plantas medicinales utilizadas en comunidades rurales del Chubut, Patagonia-Argentina . BLACPMA 3:5862.

GONZALO, J. 2002. La búsqueda de la salud perdida: los otros terapeutas. Scripta Ethnologica 14: 81131.

HERMANN P., V. CAMBI, E. BONTTI, M. HAUSSAIRE \& D. SÁNCHEZ. 2001. El uso de plantas medicinales en Bahía Blanca (Parte II). Bol.
Soc. Argent. Bot. 36(1-2):161-170.

HERNANDEZ M., S. CIVITELLA \& V. ROSATO. 2010. Uso medicinal popular de plantas y líquenes de la Isla Paulino, Provincia de Buenos Aires, Argentina. BLACPMA 9:258-268.

HILGERT, N. 2001. Plants used in home medicine in the Blanco River basin, in northwestern Argentina subtropical montane forests. Journal of Ethnopharmacology 76: 11-34.

2009. La salud en las Yungas. ¿Cuales son los principales problemas según la medicinal tradicional y la formal? En: Vignale N. \& Pochettino M. (eds.) Avances sobre plantas medicinales andinas. RISAPRET/CYTED. S. S. de Jujuy.

\& G. GIL .2006. Medicinal plants of the Argentine Yungas plants of Las Yungas biosphere reserve, Northwest of Argentina, used in health care. Biodiversity and Conservation 15:2565-2594

\& G. GIL. 2007. Reproductive medicine in northwest Argentina: traditional and institutional systems. Journal of Ethnobiology and Ethnomedicine 3:19 - 34

— \& G. GIL. 2008. Los cambios de uso del ambiente y la medicina herbolaria. Estudio de caso en Yungas argentinas. BLACPMA 7: 130-140.

—. M. HIGUERA \& M. KRISTENSEN. 2010. La medicina herbolaria en el coontexto urbano. Estudio de caso en un barrio de la ciudad de Tandil, Argentina. BLACPMA 9: 177-190.

IDOYAGA MOLINA A. 1976. Aproximación hermenéutica a las nociones de concepción, gravidez y alumbramiento entre los pilagá del Chaco Central. Scripta Ethnologica 4 (2): 78-98.

- 1978. Contribución al estudio del proceso de gestación, aborto y alumbramiento entre los Mataco costaneros. Scripta Ethnologica 5 (2): 143-155.

- 1999. Sexualidad Reproducción y aborto. Nociones y prácticas de mujeres indígenas y campesinas de Argentina. CAEA, CONICET. Buenos Aires

—. 2000 .La calidad de las prestaciones de salud y el punto de vista del ususario en un contexto de medicinas múltiples. Scripta ethnologica 22: 21-85.

_ 2001. Lo sagrado en las terapias de las medicinas tradicionales del NOA y Cuyo. Scripta ethnologica 23:9-75.

JIMENEZ DE PUPARELLI, D. 1984. Función de la medicina popular en la comunidad entrerriana y su relación con la Medicina oficial. En: Cultura tradicional del área del Paraná Medio, Buenos Aires: Fundación Bracht Editores. Instituto Nacional de Antropología. Buenos Aires.

KELLER, H. A. 2007 Notas sobre medicina y magia entre los guaraníes de Misiones, Argentina, un enfoque etnobotánico. Suplemento Antropológico de la Universidad Católica de Asunción XLII (2): 345-384.

KELLER, H. \& H. ROMERO. 2006. Plantas medicinales 
utilizadas por campesinos del área de influencia de la Reserva Yabotí (Misiones, Argentina). Bonplandia 15: 125-141.

LADIO, A., LOZADA, M., \& WEIGANT, M. 2007. Comparison of traditional wild plant knowledge between aboriginal communities inhabiting in arid and forest environments in Patagonia, Argentina. Journal of Arid Environments 69: 695-715.

LA TORRE-CUADROS, M. 2008. Ciento doce años de investigación científica sobre las etnias de la Amazonia Peruana. BLACMA 7 (3), 171-179.

LOZADA, M., A. LADIO \& M. WEIGANT. 2006. Cultural transmission of ethnobotanical knowledge in a rural community of Nortwestern Patagonia, Argentina. Economy Botany 60: 374-385.

LOZANO, P.1873. Historia de la Conquista del Paraguay, Río de la Plata y Tucumán, Tomos 1 a V. Imprenta popular Buenos Aires.

MARTINEZ CROVETTO, R. 1968a. Nombres de plantas y su utilidad, según los indios onas de Tierra del Fuego. Estudios Etnobotánicos 1V, Etnobiologica vol.n ${ }^{\circ}$ 3, Facultad de Agronomía y Veterinaria, Universidad Nacional del Nordeste, Corrientes.

1968 b. Nombres de las plantas y su utilidad según los indios araucano-pampas del oeste de Buenos Aires (República Argentina). Estudios Etnobotánicos III, Etnobiologica vol.n 12, Facultad de Agronomía y Veterinaria, Universidad Nacional del Nordeste, Corrientes.

_- 1981. Plantas utilizadas en la medicina en el NO de Corrientes. Miscelanea 69, Fundación Miguel Lillo, Ministerio de Cultura y Educación, Tucumán.

MARTINEZ, G. 2002. Conocimiento de la flora de interés etnobotánico entre estudiantes del Valle de Paravachasca, Córdoba (Argentina). Parodiana, 12 (1-12), 35-62.

_ 2005. Recolección y Comercialización de Plantas Medicinales en el Departamento Santa María, Provincia de Córdoba, Argentina. Acta Farm. Bonaerense 24 (4): 575-84.

- 2007a. Medicinal plants used by the 'Criollos' of Calamuchita (Córdoba, Argentina) to treat blood, cardiovascular and neuroendocrinous diseases. Journal Of Herbs, Spices \& Medicinal Plants, 13: 22-54.

2007b. La farmacopea natural en la salud maternoinfantil de los tobas del río Bermejito. Kurtziana 33: 39-63.

- 2007c. "Vienen buscando la vida" (Ilotaique nachaalataxac): Las trayectorias terapéuticas de los tobas del Río Bermejito (Chaco). In: A. Idoyaga Molina (ed.). Los caminos terapéuticos y los rostros de la diversidad. Tomo I. CAEA - IUNA, Buenos Aires, pp. 401-424

. 2008a. Tradicional practices, beliefs and uses of medicinal plant in relation to the maternal-infant health of the Criollo woman in Central Argentina. Midwifery 24 (4): 490-502.

- 2008b. La farmacopea natural y el tratamiento de las afecciones de la piel en la medicina tradicional de los campesinos de las sierras de Córdoba, Argentina. Dominguezia 24 (1): 27-46.

. 2010 a. Los remedios naturales en la prevención y cuidado de la salud oral de los tobas del Chaco Central (Argentina). BLACPMA 9(2): 109-122

—_. 2010 b. Los criterios terapéuticos en la farmacopea natural de los Tobas bermejeños del Chaco central (Argentina). En: Pochettino, M.A., Ladio, A. \& Arenas, P. (eds). 2010. Tradiciones y transformaciones en Etnobotánica. CYTEDPrograma Iberoamericano Ciencia y Tecnología para el Desarrollo- Editor

MARTINEZ, G. \& A. PLANCHUELO 2003. La medicina tradicional de los criollos campesinos de Paravachasca y Calamuchita, Córdoba (Argentina). Scripta Ethnológica 15: 83-116.

MARTINEZ, G., A.M. PLANCHUELO, E. FUENTES \& M. OJEDA, 2004. A numeric index to establish conservation priorities for medicinal plants in the Paravachasca Valley, Córdoba, Argentina. Biodiversity and Conservation 15: 1-19.

MARTINEZ, G., M. SATO \& M. OJEDA. 2009. Native Medicinal Plants used in the Ethnomedicine of the Córdoba Hills in Central Argentina: Relevance and Interest for Primary Health Care and Conservation. En: Varela A. \& J. Ibañez. Medicinal Plants: Classification, Biosynthesis and Pharmacology. Nova Publishers Edits. New York.

MARTINEZ, M., M. POCHETTINO \& A. CORTELLA. 2004. Environment and illness in the Calchaquí Valley (Salta, Argentina): phytotherapy for osteoarticular and cardio-circulatory diseases. Journal of Ethnopharmacology 95: 317-327.

MENSEGUEZ, P., GALETTO L. y A. ANTON 2007. El uso de las plantas medicinales en la población campesina de El Puesto (Córdoba, Argentina) Kurtziana 33 (1): 89-102.

MOLARES, S. \& A. LADIO .2008. Plantas medicinales en una comunidad Mapuche del NO de la Patagonia Argentina: clasificación y percepciones organolépticas relacionadas con su valoración. BLACPMA, 7 (3): 141 -148

MONTENEGRO, P. 1945 [1710]. Materia Médica Misionera. Biblioteca Nacional, Buenos Aires.

MOREAU, D. 2009. El uso de las plantas medicinales en la provincia de Misiones (Argentina) y su valorización como recurso en la atención primaria de la salud: exploración de una alternativa de conservación del bosque atlántico del Alto Paraná. Estudio de caso en una población campesina de Comandante Andresito, Misiones, Argentina. Fundación Vida Silvestre Argentina.

MUIÑO, W. 2010. Ethnobotanical Study of the 
Rural Population of the West of the Pampa Plain (Argentina). Ethnobotany Research \& Applications 8:219-231

MÜLLER, F. (S.V.D.) 1989. Etnografía de los guaraníes del Alto Paraná. CAEA Editorial, Buenos Aires, 133 pp.

NIETO, M. 2005. Remedios para el imperio. La Búsqueda de plantas medicinales. Universidad de Los Andes Departamento de Historia, Colombia. Disponible en: http://historiadelaciencia-nieto. uniandes.edu.co/index.html

NUÑEZ C. \& J.J. CANTERO. 2000. Las plantas medicinales del sur de la Provincia de Córdoba. Editorial de la Fundación de la Universidad Nacional de Río Cuarto, Córdoba, Argentina.

OCHOA, J., A. LADIO \& LOZADA, M. 2010. Aspectos ecológicos y socioculturales asociados al uso de plantas silvestres en la población rural de Arroyo Las Minas (Río Negro, Argentina). En: Pochettino, M.A., Ladio,A. \& Arenas, P. (eds). 2010. Tradiciones y transformaciones en Etnobotánica. CYTEDPrograma Iberoamericano Ciencia y Tecnología para el Desarrollo- Editor. Ecológicos

PAUCKE, F.1944. Hacia allá y para acá (una estancia con los indios mocovíes, 1749-1767). Tomo 111, segunda parte. Publicación $n^{\circ} 349$. Universidad Nacional de Tucumán, Publicaciones especiales del Instituto de Antropología (V), en colaboración con la Institución Cultural Argentino-Germana, Publicación n ${ }^{\circ} 11$, Buenos Aires.

PIRONDO, A., J.COULLERI, H. KELLER \& M. FERRUCCI.2011. Influencia de factores externos sobre la comercialización de plantas medicinales en un medio urbano: el caso de criollos e indígenas en Corrientes, Argentina. BLACPMA 10: 553-569.

POCHETTINO, M.L, M.R MARTÍNEZ, B. ITTEN \& M.ZUCARO. 1997. Las plantas medicinales como recurso terapéutico en una población urbana: estudio etnobotánico en Hernández (Pdo. La Plata, Prov. Buenos Aires, Argentina). Parodiana 10:141152.

POCCHETTINO, M.L. 2007. Recolección y comercialización de plantas silvestres en dos comunidades Mbya-Guaraní (Misiones, Argentina). Kurtziana 33 (1): 27-38.

POCCHETTINO, M.L., ARENAS, P., SÁNCHEZ,
D. \& R. CORREA. 2008. Conocimiento botánico tradicional, circulación comercial y consumo de plantas medicinales en un área urbana de Argentina. BLACPMA 7 (3): 141 -148

REMORINI, C.\& A. SY, 2002. Las sendas de la imperfección (Tape Rupa Reco Achy). Una aproximación etnográfica a las nociones de salud y enfermedad en comunidades Mbyá. Scripta Ethnologica XXIV: 133-147.

RIO Y ACHAVAL, 1905. Geografía Física de Córdoba. Volumen II. Gobierno de la Provincia de Córdoba, Argentina.

SANCHEZ LABRADOR, J. 1910.El Paraguay católico, 1 y 1l. Ed. Coni, Buenos Aires.

SCARPA, G.2002. Plantas empleadas contra transtornos digestivos en la medicina tradicional criolla del Chaco Noroccidental. Dominguezia 18: 36-50.

- 2004. Medicinal plants used by the Criollos of Northwestern Argentine Chaco. Journal of Ethnopharmacology 91(1): 115-135.

—. 2005. El síndrome cálido-fresco en la medicina popular criolla del Chaco argentino. Revista de Dialectología y Tradiciones Populares 59: 5-29.

. 2008. Hacerse "mala sangre" en el Chaco argentino: Etiologías, diagnóstico y terapéutica folk. Analecta Historico Medica Supl. 1, 6: 191-196.

—. 2009. Etnobotanica médica de los indígenas chorotes y su comparación con los criollos del chaco semiárido (Argentina). Darwiniana 47: 92-107.

SCHULZ, A. 1997. Algunas plantas usuales del noroeste argentino. Parodiana 10:211-241.

TRILLO, C. 2010. Valoración del bosque y conocimiento de las plantas silvestres por parte de los pobladores de las Sierras de Guasapampa, Noroeste de la Provincia de Córdoba. Tesis Doctoral, Universidad Nacional de Córdoba

—_, ARIAS TOLEDO, B., GALETTO, L. \& COLANTONIO, S. 2010 Persistence of the use of medicinal plants in rural communities of the Western Arid Chaco (Cordoba, Argentina). The Open Complementary Medicine Journal 2:80-89.

ZAMUDIO, F., M. KUJAWSKA \& N. HILGERT. 2010. Honey as medicinal and food resource. Comparison between Polish and multiethnic settlements of the Atlantic Forest, Misiones, Argentina. The Open Complementary Medicine Journal 2: 58-73.

Original recibido el 21 de noviembre de 2011; aceptado el 15 de diciembre de 2011. 
